PROCEEDINGS OF THE

AMERICAN MATHEMATICAL SOCIETY

Volume 138, Number 2, February 2010, Pages 725-736

S 0002-9939(09)10117-X

Article electronically published on October 9, 2009

\title{
COMPLETE ISOMORPHIC CLASSIFICATIONS OF SOME SPACES OF COMPACT OPERATORS
}

\author{
ELÓI MEDINA GALEGO
}

(Communicated by Nigel J. Kalton)

\begin{abstract}
This paper is a continuation and a complement of our previous work on isomorphic classification of some spaces of compact operators. We improve the main result concerning extensions of the classical isomorphic classification of the Banach spaces of continuous functions on ordinals. As an application, fixing an ordinal $\alpha$ and denoting by $X^{\xi}, \omega_{\alpha} \leq \xi<\omega_{\alpha+1}$, the Banach space of all $X$-valued continuous functions defined in the interval of ordinals $[0, \xi]$ and equipped with the supremum, we provide complete isomorphic classifications of some Banach spaces $\mathcal{K}\left(X^{\xi}, Y^{\eta}\right)$ of compact operators from $X^{\xi}$ to $Y^{\eta}, \eta \geq \omega$.

It is relatively consistent with ZFC (Zermelo-Fraenkel set theory with the axiom of choice) that these results include the following cases:

1. $X^{*}$ contains no copy of $c_{0}$ and has the Mazur property, and $Y=c_{0}(J)$ for every set $J$.

2. $X=c_{0}(I)$ and $Y=l_{q}(J)$ for any infinite sets $I$ and $J$ and $1 \leq q<\infty$.

3. $X=l_{p}(I)$ and $Y=l_{q}(J)$ for any infinite sets $I$ and $J$ and $1 \leq q<p<\infty$.
\end{abstract}

\section{Introduction AND STATEMENT OF the MAIN RESUlT}

We mostly use standard Banach space notation as may be found in 13 . In particular, if $K$ is a compact Hausdorff space and $X$ is a Banach space, by $C(K, X)$ we will denote the Banach space of all $X$-valued continuous functions defined on $K$ and equipped with the supremum norm. As in [1, when $K$ is the interval of ordinals $[0, \xi]$ endowed with the order topology, this space will also be indicated by $X^{\xi}$. We write $X \sim Y$ when the Banach spaces $X$ and $Y$ are isomorphic. The cardinality of a set $\Gamma$ will be denoted by $|\Gamma|$ and the cardinality of an ordinal $\xi$ by $\bar{\xi}$. As usual, for a fixed ordinal $\alpha, \omega_{\alpha}$ stands for the first ordinal of cardinality $\aleph_{\alpha}$. We also write $\omega=\omega_{0}$.

The main goal of the present paper is to complete the partial isomorphic classifications of some Banach spaces of compact operators recently obtained in [10] and 22. In order to do this, we first prove Theorem 1.1, which is a refinement of [10, Theorem 1.1]. This result is an extension of the classical isomorphic classification of the $\mathbb{R}^{\xi}$ spaces accomplished by Bessaga and Pełczyński [1, in the case where

Received by the editors March 5, 2009, and, in revised form, July 10, 2009.

2000 Mathematics Subject Classification. Primary 46B03, 46B25; Secondary 47B10.

Key words and phrases. Isomorphic classifications of spaces of continuous functions, compact operators.

The author would like to thank the referee for several helpful comments and suggestions which have been incorporated into the current version of the paper.

(C)2009 American Mathematical Society Reverts to public domain 28 years from publication 
$\omega \leq \alpha<\omega_{1}$; Semadeni [23], in the case where $\omega_{1}<\alpha \leq \omega_{1} \omega$; Labbé 17], in the case where $\omega_{1} \omega<\alpha<\omega_{1}^{\omega}$; and independently Kislyakov [16] and Gul'ko and Os'kin [12, in the general case.

Theorem 1.1. Let $X$ be a Banach space having the Mazur Property and containing no copy of $c_{0}, \Gamma$ a set, $\alpha$ an initial ordinal and $\xi \leq \eta$ two infinite ordinals.

(1) If $c_{0}(\Gamma, X)^{\xi} \sim c_{0}(\Gamma, X)^{\eta}$, then $\bar{\xi}=\bar{\eta}$.

(2) Suppose $\bar{\xi}=\bar{\eta}=\bar{\alpha}$ and assume that $\alpha=\omega$, or $\alpha$ is a singular ordinal, or $\alpha$ is a nondenumerable regular ordinal with $\alpha^{2} \leq \xi$. Then $c_{0}(\Gamma, X)^{\xi} \sim$ $c_{0}(\Gamma, X)^{\eta}$ if and only if $\eta<\xi^{\omega}$.

(3) Suppose that $\alpha$ is a nondenumerable regular ordinal, $\xi, \eta \in\left[\alpha, \alpha^{2}\right]$ and let $\xi^{\prime}, \eta^{\prime}, \gamma$ and $\delta$ be ordinals such that $\xi=\alpha \xi^{\prime}+\gamma, \eta=\alpha \eta^{\prime}+\delta, \xi^{\prime}, \eta^{\prime} \leq \alpha$ and $\gamma, \delta<\alpha$. Then $c_{0}(\Gamma, X)^{\xi} \sim c_{0}(\Gamma, X)^{\eta}$ if and only if $|\Gamma| \bar{\xi}^{\prime}<\aleph_{0}$ and $X^{|\Gamma| \bar{\xi}^{\prime}} \sim X^{|\Gamma| \bar{\eta}^{\prime}}$ or $\aleph_{0} \leq|\Gamma| \bar{\xi}^{\prime}=|\Gamma| \bar{\eta}^{\prime}$.

(4) Suppose that $\alpha$ is a nondenumerable regular ordinal and $\alpha \leq \xi<\alpha^{2} \leq \eta$. Then $c_{0}(\Gamma, X)^{\xi} \nsim c_{0}(\Gamma, X)^{\eta}$.

We recall that a Banach space $X$ is said to have the Mazur Property (in short, MP) if every element of $X^{* *}$ which is sequentially weak* continuous is weak* continuous and thus is an element of $X$. Such spaces were investigated in [6] and [15], and sometimes they are also called d-complete [18] or $\mu \mathrm{B}$-spaces [24].

Remark 1.2. First of all, notice that the sufficiency of the statements (2) and (3) of Theorem 1.1 follows immediately from the isomorphic classification of the spaces $\mathbb{R}^{\xi}$, with $\xi \geq \omega,\left[16\right.$, Theorem 1] and [16, Theorem 2]. Because $X^{\xi}$ is isomorphic to the injective tensor product $X \hat{\otimes} \mathbb{R} \xi$ and

$$
c_{0}(\Gamma, X)^{\xi} \sim c_{0}(\Gamma, X) \hat{\hat{\otimes}} \mathbb{R}^{\xi} \sim c_{0}(\Gamma) \hat{\hat{\otimes}} X \hat{\hat{\otimes}} \mathbb{R}^{\xi} \sim c_{0}(\Gamma) \hat{\hat{\otimes}} X^{\xi},
$$

hence for any $X, \Gamma$ and $\xi, \eta$, we have

$$
\mathbb{R}^{\xi} \sim \mathbb{R}^{\eta} \Rightarrow X^{\xi} \sim X^{\eta} \Rightarrow c_{0}(\Gamma, X)^{\xi} \sim c_{0}(\Gamma, X)^{\eta} .
$$

Further, in some cases the inverse implication of (1) holds. More precisely, Theorem 1.1 implies that the inverse of implication (1) holds if $X$ has MP, does not contain a copy of $c_{0}, \xi, \eta$ are infinite ordinals with $\xi \leq \eta$ and the cardinality of $\xi$ is either countable or singular or it is a regular ordinal $\alpha$ with $\xi \geq \alpha^{2}$. The only case when $\Gamma$ and $X$ really enter is that described in the assertion (3).

To present some applications of the above theorem, we need to recall some results of the set theory based on the axioms of Zermelo-Fraenkel and the axiom of choice. Let $m_{r}$ denotes the least real-valued measurable cardinal. It is well known that the existence of measurable cardinals cannot be proved in ZFC [14, pages 106 and 108]. On the other hand, it is relatively consistent with ZFC that measurable cardinals do not exist [11, Theorem 4.14, page 972].

So it is relatively consistent with ZFC that Corollary 1.3 and Remarks 1.6 and 1.7 provide a complete isomorphic classification of the considered spaces.

Corollary 1.3. Let $\xi, \eta, \lambda$ and $\mu$ be ordinals with $\omega \leq \xi \leq \eta, \aleph_{0} \leq \bar{\lambda}=\bar{\mu}<m_{r}, \alpha$ an initial ordinal, $\Gamma$ a set and $X$ a Banach space such that $X^{*}$ contains no copy of $c_{0}$ and has $M P$.

(1) If $\mathcal{K}\left(X^{\lambda}, c_{0}(\Gamma)^{\xi}\right) \sim \mathcal{K}\left(X^{\mu}, c_{0}(\Gamma)^{\eta}\right)$, then $\bar{\xi}=\bar{\eta}$. 
(2) Suppose $\bar{\xi}=\bar{\eta}=\bar{\alpha}$ and assume that $\alpha=\omega$, or $\alpha$ is a singular ordinal, or $\alpha$ is a nondenumerable regular ordinal with $\alpha^{2} \leq \xi$. Then $\mathcal{K}\left(X^{\lambda}, c_{0}(\Gamma)^{\xi}\right) \sim$ $\mathcal{K}\left(X^{\mu}, c_{0}(\Gamma)^{\eta}\right)$ if and only if $\eta<\xi^{\omega}$.

(3) Suppose that $\alpha$ is a nondenumerable regular ordinal, $\xi, \eta \in\left[\alpha, \alpha^{2}\right]$ and let $\xi^{\prime}, \eta^{\prime}, \gamma, \delta$ be ordinals such that $\xi=\alpha \xi^{\prime}+\gamma, \eta=\alpha \eta^{\prime}+\delta, \xi^{\prime}, \eta^{\prime} \leq \alpha$ and $\gamma, \delta<\alpha$. Then $\mathcal{K}\left(X^{\lambda}, c_{0}(\Gamma)^{\xi}\right) \sim \mathcal{K}\left(X^{\mu}, c_{0}(\Gamma)^{\eta}\right)$ if and only if $|\Gamma| \bar{\xi}^{\prime}<\aleph_{0}$ or $\aleph_{0} \leq|\Gamma| \bar{\xi}^{\prime}=|\Gamma| \bar{\eta}^{\prime}$.

(4) Suppose that $\alpha$ is a nondenumerable regular ordinal and $\alpha \leq \xi<\alpha^{2} \leq \eta$. Then $\mathcal{K}\left(X^{\lambda}, c_{0}(\Gamma)^{\xi}\right) \nsim \mathcal{K}\left(X^{\mu}, c_{0}(\Gamma)^{\eta}\right)$.

Proof. Suppose first that $Y$ is any Banach space. Since $c_{0}(\Gamma)^{\xi}$ has the approximation property [5, Corollary 5, page 242] and [5, Example 11, page 245], it follows from [3. Proposition 5.3] and [5, Example 6, page 224] that

$$
\mathcal{K}\left(Y, c_{0}(\Gamma)^{\xi}\right) \sim Y^{*} \hat{\hat{\otimes}} c_{0}(\Gamma)^{\xi} \sim Y^{*} \hat{\hat{\otimes}} c_{0}(\Gamma) \hat{\hat{\otimes}} \mathbb{R}^{\xi} \sim c_{0}\left(\Gamma, Y^{*}\right) \hat{\hat{\otimes}} \mathbb{R}^{\xi} \sim c_{0}\left(\Gamma, Y^{*}\right)^{\xi} .
$$

Then, taking $Y=X^{\lambda}$ and using the fact that $\left.\left(X^{\lambda}\right)^{*}=(C[0, \lambda], X)\right)^{*} \sim l_{1}\left([0, \lambda], X^{*}\right)$, we see that

$$
\mathcal{K}\left(X^{\lambda}, c_{0}(\Gamma)^{\xi}\right) \sim c_{0}\left(\Gamma, l_{1}\left([0, \lambda], X^{*}\right)\right)^{\xi} .
$$

By a standard gliding hump argument we can prove that $l_{1}\left([0, \lambda], X^{*}\right)$ contains no copy of $c_{0}$; see for instance [2]. Moreover, since $X^{*}$ has MP, $l_{1}\left([0, \lambda], X^{*}\right)$ also has MP because $\bar{\lambda}<m_{r}$ [15, Theorem 3.1]. Then the result follows directly from Theorem 1.1.

Remark 1.4. Observe that, as is very easily seen, by 9 , Theorem 2.3], if $\mathcal{K}\left(\mathbb{R}^{\lambda}, \mathbb{R}^{\xi}\right)$ $\sim \mathcal{K}\left(\mathbb{R}^{\mu}, \mathbb{R}^{\eta}\right)$ for some ordinals $\xi, \eta, \lambda$ and $\mu$, then $\bar{\lambda}=\bar{\mu}$. Therefore, Corollary 1.3 furnishes a complete isomorphic classification of the spaces $\mathcal{K}\left(\mathbb{R}^{\xi}, \mathbb{R}^{\eta}\right)$, where $\xi \geq \omega$ and $\eta \geq \omega$. The case $\omega \leq \xi=\eta<\omega_{1}$ is due to C. Samuel [22], and the case $\omega \leq \xi<\omega_{1}$ and $\eta \geq \omega$ was obtained in [10] under the Continuum Hypothesis.

Remark 1.5. (a) We stress that when $\Gamma$ is a singleton, $c_{0}(\Gamma, X) \sim X$, and thus Theorem 1.1 provides in particular the isomorphic classification of the spaces $X^{\xi}$, with $\xi \geq \omega$, whenever $X$ has MP and contains no copy of $c_{0}$.

(b) If $X$ is in addition isomorphic to its square $X^{2}$, then the third statement of Theorem 1.1 is reduced to $X^{\xi} \sim X^{\eta}$ if and only if $\bar{\xi}^{\prime}<\aleph_{0}$ or $\aleph_{0} \leq \bar{\xi}^{\prime}=\bar{\eta}^{\prime}$.

Remark 1.6. Fix $1 \leq p, q<\infty, \alpha$ an ordinal, $I$ and $J$ infinite sets satisfying $\aleph_{\alpha}|I|<m_{r}$. As we have already mentioned above, $c_{0}(\Gamma)^{\xi}$ has the approximation property for every ordinal $\xi$. So, for every ordinal $\eta \geq \omega$, we infer that

$$
\mathcal{K}\left(c_{0}(I)^{\xi}, l_{q}(J)^{\eta}\right) \sim l_{1}\left([0, \xi], l_{1}(I)\right) \hat{\hat{\otimes}} l_{q}(J)^{\eta} \sim\left(l_{1}([0, \xi] \times I) \hat{\hat{\otimes}} l_{q}(J)\right)^{\eta} .
$$

Moreover, the spaces $\left.l_{1}([0, \xi] \times I) \hat{\hat{\otimes}} l_{q}(J)\right)$ contain no copy of $c_{0}$ [21, Main Theorem].

Now if we suppose that $\bar{\xi}=\aleph_{\alpha}$, then $\bar{\xi}|I|<m_{r}$ and consequently the spaces $\left.l_{1}([0, \xi] \times I) \hat{\hat{\otimes}} l_{q}(J)\right)$ have MP $([15$, Theorem 3.1] and [15, Corollary 5.2.1]). Thus by Remark 1.5(b) we get the isomorphic classification of the spaces $\mathcal{K}\left(c_{0}(I)^{\xi}, l_{q}(J)^{\eta}\right)$ for $\xi$ in the interval $\left[\omega_{\alpha}, \omega_{\alpha+1}[\right.$ and $\eta \geq \omega$.

Remark 1.7. Fix $1 \leq p, q<\infty$ with $p>q, \alpha$ an ordinal, $I$ and $J$ infinite sets satisfying $\aleph_{\alpha}<m_{r}$. Since $l_{p}(I)^{\xi}$ has the approximation property for every ordinal $\xi$ [5, Example 11, page 245], we deduce that, for every $\eta \geq \omega$,

$$
\mathcal{K}\left(l_{p}(I)^{\xi}, l_{q}(J)^{\eta}\right) \sim l_{1}\left([0, \xi], l_{p^{\prime}}(I)\right) \hat{\hat{\otimes}} l_{q}(J)^{\eta} \sim\left(l_{1}\left([0, \xi], l_{p^{\prime}}(I)\right) \hat{\hat{\otimes}} l_{q}(J)\right)^{\eta},
$$


where $1 / p+1 / p^{\prime}=1$. Furthermore, by the classical Pitt's compactness theorem [19, Proposition 2.c.3], every linear operator from $c_{0}$ or $l_{p}$ into $l_{q}$ is compact. So by [4. Proposition 6] the same is true for every linear operator from $c_{o}\left(l_{p}\right)$ into $l_{q}$. Then by [25, Corollary 14] we conclude that the spaces $l_{1}\left([0, \xi], l_{p^{\prime}}(I)\right) \hat{\otimes} l_{q}(J)$ contain no copy of $c_{0}$.

On the other hand, if we assume that $\bar{\xi}=\aleph_{\alpha}$, then according to [15, Theorem 3.1] and [15. Corollary 5.2.1], we have that the spaces $l_{1}\left([0, \xi], l_{p^{\prime}}(I)\right) \hat{\hat{\otimes}} l_{q}(J)$ have MP. Thus again by Remark 1.5(b) we obtain the isomorphic classification of the spaces $\mathcal{K}\left(l_{p}(I)^{\xi}, l_{q}(J)^{\eta}\right)$ for $\xi$ in the interval $\left[\omega_{\alpha}, \omega_{\alpha+1}[\right.$ and $\eta \geq \omega$.

\section{SOME PRELIMINARY RESULTS}

In this section, we introduce Definition 2.2, which allows us to strengthen earlier results of [7; see Lemmas 2.4 and 2.6 and Propositions 2.7 and 2.8. In contrast to the analogous results of [7, these new results need no additional hypothesis on the space $X^{*}$. As a consequence, we generalize some results of [10; see Propositions 2.13 and 3.2 and Lemma 3.1, which, in turn, will be useful in the proof of Theorem 1.1.

We begin by recalling a few necessary definitions that have been introduced in [7. Let $\gamma$ be an ordinal. A $\gamma$-sequence in a set $A$ is a function $f:[1, \gamma[\rightarrow A$ and will be denoted by $\left(x_{\theta}\right)_{\theta<\gamma}$. If $A$ is a topological space and $\beta$ is an ordinal, we will say that the $\gamma$-sequence $\left(x_{\theta}\right)_{\theta<\gamma}$ is $\beta$-continuous if for every $\beta$-sequence of ordinals $\left(\theta_{\xi}\right)_{\xi<\beta}$ of $[0, \gamma]$ which converges to $\theta_{\beta}$ when $\xi$ converges to $\beta$, we have that $x_{\theta_{\xi}}$ converges to $x_{\theta_{\beta}}$.

Let $X$ be a Banach space, $\alpha$ an ordinal number and $\varphi$ a cardinal number. By $X_{\alpha}^{\varphi}$ we will denote the space of all $x^{* *} \in X^{* *}$ having the following property: for every set $B$ with $|B|=\varphi, \beta<\alpha$ and $B$-family $x^{b}=\left(x_{\xi}^{*}(b)\right)_{\xi<\beta}, b \in B$, of $\beta$-sequences of $X^{*}$ such that there exists $M \in \mathbb{R}$ with $\left\|x_{\xi}^{*}(b)\right\| \leq M$ for every $b \in B$ and $\xi<\beta$ and such that $x_{\xi}^{*}(b)(x) \stackrel{\xi \rightarrow \beta}{\longrightarrow} 0, \forall x \in X$, uniformly in $b$, we have $x^{* *}\left(x_{\xi}^{*}(b)\right) \stackrel{\xi \rightarrow \beta}{\longrightarrow} 0$ uniformly in $b$.

Remark 2.1. Let $X, \alpha, \beta, \varphi, B, x^{b}=\left(x_{\xi}^{*}(b)\right)_{\xi<\beta}$ and $M$ be as above. Suppose that $\left(x_{n}^{* *}\right)_{n<\omega}$ is an $\omega$-sequence in $X_{\alpha}^{\varphi}$ converging to $x^{* *}$ in $X^{* *}$. Since

$$
\left|x^{* *}\left(x_{\xi}^{*}(b)\right)\right| \leq\left\|x_{n}^{* *}-x^{* *}\right\| M+\left|x_{n}^{* *}\left(x_{\xi}^{*}(b)\right)\right|,
$$

for every $n<\omega, b \in B$ and $\xi<\beta$, it follows that $X_{\alpha}^{\varphi}$ is a closed subspace of $X^{* *}$.

Clearly $c X \subset X_{\alpha}^{\varphi}$, where $c X$ is the canonical image of $X$ in $X^{* *}$. Observe also that if $X$ has MP, then $X_{\alpha}^{\varphi}=c X$.

We now come to the central definition of this paper:

Definition 2.2. Let $X$ be a Banach space and $\alpha$ a nondenumerable regular ordinal. Then we set

$$
[X]_{\alpha}=\bigcap_{\varphi<\bar{\alpha}} X_{\alpha}^{\varphi} .
$$

Remark 2.3. It is readily verified that if $X$ and $Y$ are isomorphic Banach spaces, then

$$
\frac{[X]_{\alpha}}{c X} \sim \frac{[Y]_{\alpha}}{c Y} .
$$

From now on, $X \hookrightarrow Y$ means that the Banach space $Y$ contains a copy of the Banach space $X$. The next lemma was inspired by [16, Lemma 1.4]. 
Lemma 2.4. Let $X$ be a closed subspace of the Banach space $Y$ and $\alpha$ a nondenumerable regular ordinal. Then

$$
\frac{[X]_{\alpha}}{c X} \hookrightarrow \frac{[Y]_{\alpha}}{c Y} .
$$

Proof. Let $i$ be the identity embedding of $X$ into $Y$. It is easy to see that $i^{* *}\left([X]_{\alpha}\right) \subset$ $[Y]_{\alpha}$. Thus we can consider the quotient operator $T: \frac{[X]_{\alpha}}{c X} \rightarrow \frac{[Y]_{\alpha}}{c Y}$. We will show that $T$ is an isomorphism onto its image. Notice that it suffices to prove that there exists no $F \in[X]_{\alpha}$ such that $d(F, c X)>1$ and $d\left(i^{* *}(F), c Y\right)<1 / 3$. Suppose then that such an $F$ exists. Fix $y \in Y$ satisfying $\left\|i^{* *}(F)-c y\right\|<1 / 3$. Then we can check that $d(y, i(X))>2 / 3$. Next, by the Hahn-Banach theorem, pick $y^{*} \in Y^{*}$ such that $\left\|y^{*}\right\|=1, y^{*}(i x)=0$ for every $x \in X$ and $\left|y^{* *}(y)\right|>2 / 3$. Therefore $i^{* *}(F)\left(y^{* *}\right)=0$ and $\left|y^{*}(y)\right|=\left|\left(c y-i^{* *}(F)\right)\left(y^{*}\right)\right| \leq\left\|c y-i^{* *}(F)\right\|<1 / 3$, which is absurd.

We also define:

Definition 2.5. Let $X$ be a Banach space, $\alpha$ a nondenumerable regular ordinal and $\gamma$ an arbitrary ordinal. By $m_{\alpha}([0, \gamma], X)$ we will denote the closed subspace of $l_{\infty}\left([0, \gamma], X^{* *}\right)$ consisting of $(\gamma+1)$-sequences $x_{\theta}^{* *}$ which are $\beta$-continuous for every $\beta<\alpha$ and such that $x_{\theta}^{* *} \in[X]_{\alpha}$ for every $\theta \leq \gamma$.

The proof of the following lemma is straightforward from the definitions.

Lemma 2.6. Let $X$ be a Banach space, $\alpha$ a nondenumerable regular ordinal, $\gamma$ an arbitrary ordinal and $g \in m_{\alpha}([0, \gamma], X)$. Then for every limit ordinal $\beta<\gamma$ there exists the limit $\tilde{g}(\beta)=\lim _{\xi \rightarrow \beta} g(\xi)$. Moreover, writing $\tilde{g}(\theta)=g(\theta)$ for every non-limit-ordinal $\theta \in[0, \gamma]$, we have that $\tilde{g}$ is continuous and $h=g-\tilde{g}$ is in $c_{0}\left([0, \gamma],[X]_{\alpha}\right)$.

The key to refining [10, Theorem 1.1] and obtaining Theorem 1.1 is the following proposition, which is an improvement of [7, Proposition 2.6]. As we will see just below, by virtue of the definition of the spaces $[X]_{\alpha}$, part of Proposition 2.7 can be proved by contradiction. This method of proof does not work when we are considering only a fixed space $X_{\alpha}^{\varphi}$ as is the case of [7, Proposition 2.6]. That is why in [7, Proposition 2.6] an additional hypothesis on the density character of $X^{*}$ was necessary.

Proposition 2.7. Let $X$ be a Banach space, $\alpha$ a nondenumerable regular satisfying $[X]_{\alpha}=c X$ and $\gamma$ an arbitrary ordinal. Then $\left[X^{\gamma}\right]_{\alpha}=\left(H^{*}\right)^{-1}\left(m_{\alpha}([0, \gamma], X)\right)$, where $H$ is the usual isometry from $l_{1}\left([0, \gamma], X^{*}\right)$ onto $\left(X^{\gamma}\right)^{*}$.

Proof. We split the proof into three parts.

- If $H^{*}(F)=\left(F_{\theta}\right)_{\theta \leq \gamma}$ with $F \in\left[X^{\gamma}\right]_{\alpha}$, then $F_{\theta} \in[X]_{\alpha}$, for every $\theta \leq \gamma$. Indeed, fix $\theta_{0} \leq \gamma$. We must show that $F_{\theta_{0}} \in X_{\alpha}^{\varphi}$ for every $\varphi<\bar{\alpha}$. Pick $\varphi<\bar{\alpha}$. Now take a set $B$ with $|B|=\varphi, \beta<\alpha$, and $x^{b}=\left(x_{\xi}^{*}(b)\right)_{\xi<\beta}, b \in B$, a $B$-family of $\beta$-sequences in $X^{*}$ such that there exists $M \in \mathbb{R}$ satisfying $\left\|x_{\xi}^{*}(b)\right\| \leq M$ for every $b \in B$ and $\xi<\beta$ and $x_{\xi}^{*}(b)(x) \stackrel{\xi \rightarrow \beta}{\longrightarrow} 0, \forall x \in X$, uniformly in $b$.

Let $u^{b}=\left(u_{\xi}^{*}(b)\right)_{\xi<\beta}$ be the $B$-family of $\beta$-sequences in $l_{1}\left([0, \gamma], X^{*}\right)$ defined by

$$
u_{\xi}^{*}(b)(\theta)= \begin{cases}x_{\xi}^{*}(b) & \text { if } \theta=\theta_{0}, \\ 0 & \text { otherwise. }\end{cases}
$$


Observe that $H\left(u_{\xi}^{*}(b)\right)(f)=x_{\xi}^{*}(b)\left(f\left(\theta_{0}\right)\right), \forall f \in X^{\gamma}$ and $F\left(H\left(u_{\xi}^{*}(b)\right)\right)=F_{\theta_{0}}\left(x_{\xi}^{*}(b)\right)$, for every $b \in B$ and $\xi<\beta$.

Moreover, since $x_{\xi}^{*}(b)\left(f\left(\theta_{0}\right)\right) \stackrel{\xi \rightarrow \beta}{\longrightarrow} 0, \forall f \in X^{\gamma}$, uniformly in $b$ and $\left\|H\left(u_{\xi}^{*}(b)\right)\right\| \leq$ $M$, for every $b \in B$ and $\xi<\beta$, it follows that $F_{\theta_{0}}\left(x_{\xi}^{*}(b)\right)=F\left(H\left(u_{\xi}^{*}(b)\right)\right) \stackrel{\xi \rightarrow \beta}{\longrightarrow} 0$, because by hypothesis $F \in\left[X^{\gamma}\right]_{\alpha} \subset\left(X^{\gamma}\right)_{\alpha}^{\varphi}$. So we are done.

- $\left[X^{\gamma}\right]_{\alpha} \subset\left(H^{*}\right)^{-1}\left(m_{\alpha}([0, \gamma], X)\right)$. By the first part of the proof it is enough to prove that if $F \in\left[X^{\gamma}\right]_{\alpha}$, then $H^{*}(F)=\left(F_{\theta}\right)_{\theta \leq \gamma}$ is $\beta$-continuous for every $\beta<\alpha$.

Indeed, let us suppose for a contradiction that there exists $\beta<\alpha$ such that $\left(F_{\theta}\right)_{\theta \leq \gamma}$ is not $\beta$-continuous. Let $\left(\theta_{\xi}\right)_{\xi<\beta}$ be a $\beta$-sequence of ordinals in $[0, \gamma]$ converging to $\beta$ when $\xi$ converges to $\beta$ such that $F_{\theta_{\xi}}$ does not converge to $F_{\theta_{\beta}}$. Then there exists $\epsilon>0$ such that for every $\eta<\beta$, there exists an ordinal $\eta<\delta(\eta)<\beta$ satisfying $\left\|F_{\theta_{\delta(\eta)}}-F_{\theta_{\beta}}\right\| \geq \epsilon$.

Therefore, for each $\eta<\beta$, there exists $x_{\delta(\eta)}^{*}$ in the unit ball of $X^{*}$ such that

$$
\left|F_{\theta_{\delta(\eta)}}\left(x_{\delta(\eta)}^{*}\right)-F_{\theta_{\beta}}\left(x_{\delta(\eta)}^{*}\right)\right| \geq \frac{\epsilon}{2} .
$$

Next we define a $\beta$-sequence $v^{\eta}=\left(v_{\xi}^{*}(\eta)\right)_{\xi<\beta}, \eta<\beta$, of $\beta$-sequences in $l_{1}\left([0, \gamma], X^{*}\right)$ by setting

$$
v_{\xi}^{*}(\eta)(\theta)= \begin{cases}x_{\delta(\eta)}^{*} & \text { if } \theta=\theta_{\xi}, \\ -x_{\delta(\eta)}^{*} & \text { if } \theta=\theta_{\beta}, \\ 0 & \text { otherwise. }\end{cases}
$$

Then, for every $f \in X^{\gamma}, \eta<\beta$ and $\xi<\beta$, since $\left\|x_{\delta(\eta)}^{*}\right\| \leq 1$, we have

$$
\left|H\left(v_{\xi}^{*}(\eta)\right)(f)\right|=\left|x_{\delta(\eta)}^{*}\left(f\left(\theta_{\xi}\right)\right)-x_{\delta(\eta)}^{*}\left(f\left(\theta_{\beta}\right)\right)\right| \leq \| f\left(\theta_{\xi}\right)-f\left(\theta_{\beta} \| .\right.
$$

Hence $H\left(v_{\xi}^{*}(\eta)\right)(f) \stackrel{\xi \rightarrow \beta}{\longrightarrow} 0, \forall f \in X^{\gamma}$, uniformly in $\eta<\beta$.

Furthermore, $\left\|H\left(v_{\xi}^{*}(\eta)\right)\right\| \leq 2$ for every $\eta<\beta$ and $\xi<\beta$. Since $F \in\left[X^{\gamma}\right]_{\alpha}$, we would have

$$
F\left(H\left(v_{\xi}^{*}(\eta)\right)\right)=F_{\theta_{\xi}}\left(x_{\delta(\eta)}^{*}\right)-F_{\theta_{\beta}}\left(x_{\delta(\eta)}^{*}\right) \stackrel{\xi \rightarrow \beta}{\longrightarrow} 0, \text { uniformly in } \eta<\beta,
$$

which is a contradiction with (2).

- $\left(H^{*}\right)^{-1}\left(m_{\alpha}([0, \gamma], X)\right) \subset\left[X^{\gamma}\right]_{\alpha}$. This part of the proof is essentially the same as the analogous part of the proof of [7, Proposition 2.6]. Fix $\varphi<\bar{\alpha}$. We must show that $\left(H^{*}\right)^{-1}\left(m_{\alpha}([0, \gamma], X)\right) \subset\left(X^{\gamma}\right)_{\alpha}^{\varphi}$. Suppose that $g \in m_{\alpha}([0, \gamma], X)$. Write $h=g-\tilde{g}$ as in Lemma 2.6. By hypothesis, $[X]_{\alpha}=c X$; therefore $\tilde{g} \in(c X)^{\gamma}=$ $H^{*}\left(c\left(X^{\gamma}\right)\right) \subset H^{*}\left(\left(X^{\gamma}\right)_{\alpha}^{\varphi}\right)$, that is, $\left(H^{*}\right)^{-1}(\tilde{g}) \in\left(X^{\gamma}\right)_{\alpha}^{\varphi}$. Then it is enough to prove that $\left(H^{*}\right)^{-1}(h) \in\left(X^{\gamma}\right)_{\alpha}^{\varphi}$.

To do this, let $B$ be a set with $|B|=\varphi, \beta<\alpha$ and a $B$-family $u^{b}=\left(u_{\xi}^{*}(b)\right)_{\xi<\beta}$, $b \in B$, of $\beta$-sequences in $l_{1}\left([0, \gamma], X^{*}\right)$ such that there exists $M \in \mathbb{R}$ satisfying $\left\|u_{\xi}^{*}(b)\right\| \leq M$, for every $b \in B, \xi<\beta$ and $H\left(u_{\xi}^{*}(b)\right)(f) \stackrel{\xi \rightarrow \beta}{\longrightarrow} 0, \forall f \in X^{\gamma}$, uniformly in $b$.

From now on, our task is to show that

$$
\left(H^{*}\right)^{-1}(h)\left(H\left(u_{\xi}^{*}(b)\right)\right)=\sum_{\lambda} h(\lambda) u_{\xi}^{*}(b)(\lambda) \stackrel{\xi \rightarrow \beta}{\longrightarrow} 0, \forall f \in X^{\gamma}, \text { uniformly in } b .
$$

To see this, let $C=\{\lambda \in[0, \gamma]: h(\lambda) \neq 0\}$ and $D=\left\{\lambda \in[0, \gamma]: u_{\xi}^{*}(b)(\lambda) \neq\right.$ 0 , for some $b \in B$ and some $\xi<\beta\}$. 
Notice that the cardinality of $D$ is smaller than $\bar{\alpha}$, because the support of each $u_{\xi}^{*}(b)$ is enumerable and $\varphi \bar{\beta}<\bar{\alpha}$. In particular, no element of $C$ is a limit point of $D$. Indeed, if there exist $c \in C, \beta_{1}<\alpha$ and a $\beta_{1}$-sequence $\left(d_{\xi}\right)_{\xi<\beta_{1}}$ in $D$ such that $d_{\xi} \stackrel{\xi \rightarrow \beta_{1}}{\longrightarrow} c$, then by the definition of $m_{\alpha}([0, \gamma], X)$, it would follow that $g\left(d_{\xi}\right) \stackrel{\xi \rightarrow \beta_{1}}{\longrightarrow} g(c)=\tilde{g}(c)$. Hence $h(c)=0$, and we would have a contradiction.

Thus each point of $E=C \cap D$ is isolated in $D$. Therefore, if $\lambda \in E$, there exists a non-limit-ordinal $\delta<\lambda$ such that $] \delta, \lambda] \cap D=\{\lambda\}$. So $u_{\xi}^{*}(b)(\theta)=0$ for every $b \in B, \xi<\beta$ and $\delta<\theta<\lambda$.

Let $x$ belong to $X$ and consider $f$ in $X^{\gamma}$ defined by $f(\theta)=x$ if $\delta<\theta \leq \lambda$, $f(\theta)=0$ otherwise. Then

$$
H\left(u_{\xi}^{*}(b)\right)(f)=\sum_{\delta<\theta \leq \lambda} u_{\xi}^{*}(b)(\theta)(f(\theta))=u_{\xi}^{*}(b)(\lambda)(x) .
$$

Hence $u_{\xi}^{*}(b)(\lambda)(x) \stackrel{\xi \rightarrow \beta}{\longrightarrow} 0, \forall x \in X$, uniformly in $b$. Observe also that $\left\|u_{\xi}^{*}(b)(\lambda)\right\| \leq$ $\left\|u_{\xi}^{*}(b)\right\| \leq M$ for every $b \in B$ and $\xi<\beta$.

Let $\varepsilon>0$ and $E_{1}=\{\lambda \in E:\|h(\lambda)\| \geq \varepsilon\}$. It follows from Lemma 2.6 that $E_{1}$ is finite. Since $h(\lambda) \in X_{\alpha}^{\varphi}$, for every $\lambda$ in $E_{1}$, we see that

$$
\sum_{\lambda \in E_{1}} h(\lambda)\left(u_{\xi}^{*}(b)\right)(\lambda) \stackrel{\xi \rightarrow \beta}{\longrightarrow} 0 \quad \text { uniformly in } b .
$$

On the other hand,

$$
\left\|\sum_{\lambda \in E} h(\lambda)\left(u_{\xi}^{*}(b)(\lambda)\right)\right\| \leq\left\|\sum_{\lambda \in E_{1}} h(\lambda) u_{\xi}^{*}(b)\right\|+\epsilon M .
$$

Consequently (3) holds.

As in [16, if $\alpha$ is a nondenumerable regular ordinal and $\gamma$ is an arbitrary ordinal, by $\Lambda_{\gamma}^{\alpha}$ we will denote the set of all ordinals from $[0, \gamma]$ with cofinality at least $\bar{\alpha}$.

Proposition 2.8. Let $\alpha$ be a nondenumerable regular ordinal, $\gamma$ an arbitrary ordinal and $X$ a Banach space satisfying $[X]_{\alpha}=c X$. Then

$$
\frac{\left[X^{\gamma}\right]_{\alpha}}{c\left(X^{\gamma}\right)} \sim \frac{m_{\alpha}([0, \gamma], X)}{(c X)^{\gamma}} \sim c_{0}\left(\Lambda_{\gamma}^{\alpha}, X\right) .
$$

Proof. The first isomorphism is an immediate consequence of Proposition 2.7 and the fact that $\left(H^{*}\right)^{-1}\left((c X)^{\gamma}\right)=c\left(X^{\gamma}\right)$. To prove the second isomorphism it suffices to apply Lemma 2.6.

We are now ready to improve [10, Lemma 2.6] when $\Gamma$ is a singleton as follows:

Lemma 2.9. Let $X$ be a Banach space having $M P, \alpha$ a nondenumerable regular ordinal. If $\mathbb{R}^{\alpha^{2}} \hookrightarrow X^{\eta}$ for some $\eta<\alpha^{2}$, then $c_{0} \hookrightarrow X$.

Proof. We distinguish two cases:

Case 1. $\eta<\alpha$. In this case, it follows from $\mathbb{R}^{\alpha} \hookrightarrow \mathbb{R}^{\alpha^{2}} \hookrightarrow X^{\eta}$ and [10, Lemma 2.3] that $c_{0} \hookrightarrow X$.

Case 2. $\alpha \leq \eta<\alpha^{2}$. Thus $\eta=\alpha \xi+\theta$ for some ordinals $\xi<\alpha$ and $\theta<\alpha$. Since $\mathbb{R}^{\eta} \sim \mathbb{R}^{\alpha \xi}$ [16, Theorem 2], we have

$$
\mathbb{R}^{\alpha^{2}} \hookrightarrow X^{\eta} \sim X \hat{\hat{\otimes}} \mathbb{R}^{\eta} \sim X \hat{\hat{\otimes}} \mathbb{R}^{\alpha \xi} \sim X^{\alpha \xi} .
$$


Let $I$ and $J$ be two sets with $|I|=\bar{\alpha}$ and $|J|=\bar{\xi}$. According to Lemma 2.4 and Proposition 2.8 we have

$$
c_{0}(I) \sim \frac{\left[\mathbb{R}^{\alpha^{2}}\right]_{\alpha}}{c\left(\mathbb{R}^{\alpha^{2}}\right)} \hookrightarrow \frac{\left[X^{\alpha \xi}\right]_{\alpha}}{c\left(X^{\alpha \xi}\right)} \sim c_{0}(J, X) .
$$

Thus by [10, Lemma 2.4] we infer that $c_{0} \hookrightarrow X$.

The following result is a generalization of [10, Lemma 2.7] when $\Gamma$ is a singleton.

Lemma 2.10. Let $\alpha$ be an initial ordinal and $\xi \leq \eta$ ordinals with $\bar{\xi}=\bar{\eta}=\bar{\alpha}$. Put $\alpha_{0}=\omega^{\omega}$ if $\alpha=\omega, \alpha_{0}=\alpha$ if $\alpha$ is a singular ordinal and $\alpha_{0}=\alpha^{2}$ if $\alpha$ is a nondenumerable regular ordinal. Suppose that $X$ is a Banach space having MP and containing no copy of $c_{0}$. If $\mathbb{R}^{\eta} \hookrightarrow X^{\xi}$ and $\alpha_{0} \leq \xi$, then $\mathbb{R}^{\eta} \hookrightarrow \mathbb{R}^{\xi}$.

Proof. We introduce two sets of ordinals:

$I_{1}=\left\{\theta: \bar{\theta}=\bar{\alpha}, \alpha_{0} \leq \theta, \mathbb{R}^{\theta} \hookrightarrow \mathbb{R}^{\gamma}, \forall \gamma<\theta\right\}$,

$I_{2}=\left\{\theta: \bar{\theta}=\bar{\alpha}, \alpha_{0} \leq \theta, \mathbb{R}^{\theta} \leadsto X^{\gamma}, \forall \gamma<\theta\right\}$.

By [10, Lemma 2.2], [10, Lemma 2.3] and Lemma 2.9, $\alpha_{0} \in I_{2}$. It suffices to proceed as in the proof of [10, Lemma 2.7] to complete the proof of this lemma.

Finally, we will state Proposition 2.13, which extends [10, Lemma 2.3]. We will need two lemmas in the course of the proof of this proposition.

Lemma 2.11. Let $\xi$ be an infinite ordinal. If $\mathbb{R}^{\xi^{\omega}} \hookrightarrow X^{\xi}$ for some Banach space $X$ having $M P$, then $c_{0} \hookrightarrow X$.

Proof. Let $\alpha$ be the initial ordinal of cardinality $\bar{\xi}$. It is convenient to consider three cases:

Case 1. $\alpha=\omega$. Assume first that $\omega^{\omega} \leq \xi$. Since $\mathbb{R}^{\xi^{\omega}} \hookrightarrow \mathbb{R}^{\xi}$ [16, Theorem 1], by Lemma 2.10, $c_{0} \hookrightarrow X$. Now suppose that $\xi<\omega^{\omega}$. Then again by [16, Theorem 1], $\mathbb{R}^{\xi} \sim \mathbb{R}^{\omega}$. Therefore $\mathbb{R}^{\omega^{\omega}} \hookrightarrow \mathbb{R}^{\xi^{\omega}} \hookrightarrow X^{\xi} \sim X \hat{\hat{\otimes}} \mathbb{R}^{\xi} \sim X \hat{\hat{\otimes}} \mathbb{R}^{\omega} \sim X^{\omega}$. Thus according to [10, Lemma 2.2], $c_{0} \hookrightarrow X$.

Case 2. $\alpha$ is a singular ordinal. Once again by [16, Theorem 1], $\mathbb{R}^{\xi^{\omega}} \hookrightarrow \mathbb{R}^{\xi}$. Hence Lemma 2.10 implies that $c_{0} \hookrightarrow X$.

Case 3. $\alpha$ is a nondenumerable regular ordinal. If $\alpha^{2} \leq \xi$, by [16, Theorem 1], $\mathbb{R}^{\xi^{\omega}} \hookrightarrow \mathbb{R}^{\xi}$. So by Lemma 2.10, $c_{0} \hookrightarrow X$. Otherwise, $\alpha \leq \xi<\alpha^{2}$. Consequently $\alpha^{2}<\alpha^{\omega} \leq \xi^{\omega}$. Therefore $\mathbb{R}^{\alpha^{2}} \hookrightarrow \mathbb{R}^{\xi^{\omega}} \hookrightarrow \mathbb{R}^{\xi}$ and by Lemma $2.9, c_{0} \hookrightarrow X$.

The framework of the proof of the next lemma was inspired by the proof of 1. Lemma 2], where Bessaga and Pełczyński obtained the complete isomorphic classification of the $\mathbb{R}^{\xi}$ spaces, with $\omega \leq \xi<\omega_{1}$.

Lemma 2.12. Let $\Gamma$ be a set, $\left(X_{\gamma}\right)_{\gamma \in \Gamma}$ a family of Banach spaces and $\eta$ an infinite ordinal.

$$
\text { If } \mathbb{R}^{\eta^{\omega}} \hookrightarrow\left(\sum_{\gamma \in \Gamma} X_{\gamma}\right)_{0} \text {, then } \mathbb{R}^{\eta} \hookrightarrow X_{\gamma_{1}} \oplus X_{\gamma_{2}} \oplus \ldots \oplus X_{\gamma_{n}} \text { for some } 1 \leq n<\omega \text {. }
$$

Proof. Suppose for contradiction that we have

$$
\mathbb{R}^{\eta^{\omega}} \hookrightarrow\left(\sum_{\gamma \in \Gamma} X_{\gamma}\right)_{0} \text { and } \mathbb{R}^{\eta} \hookrightarrow X_{\gamma_{1}} \oplus X_{\gamma_{2}} \oplus \ldots \oplus X_{\gamma_{n}}
$$


for every $1 \leq n<\omega$. Let $Y$ be a subspace of $\left(\sum_{\gamma \in \Gamma} X_{\gamma}\right)_{0}$ isomorphic to $\mathbb{R}^{\eta^{\omega}}$. Thus there exists a linear operator $T: Y \rightarrow \mathbb{R}^{\eta^{\omega}}$ and $a \in \mathbb{R}_{+}$such that

$$
\|x\| \leq\|T(x)\| \leq a\|x\|, \forall x \in Y .
$$

Fix an integer $n$ such that $n>4 a$, and let $X_{n}$ be a subspace of $\left(\sum_{\gamma \in \Gamma} X_{\gamma}\right)_{0}$ satisfying $T\left(X_{n}\right)=\mathbb{R}^{\eta^{n}}$. We next consider $\mathbb{R}^{\eta^{n}}$ embedded into $\mathbb{R}^{\eta^{\omega}}$ in such a way that the elements of $\mathbb{R}^{\eta^{n}}$ are completed by zeros on $\left(\eta^{n}, \eta^{\omega}\right]$. Pick $y_{0} \in \mathbb{R}^{\eta^{\omega}}$, the characteristic function of $\left[0, \eta^{n}\right]$, and $x_{0}=T^{-1}\left(y_{0}\right) \in X_{n}$.

There exists a finite subset $\Gamma_{1}$ of $\Gamma$ such that $\left\|x_{0}(\gamma)\right\|<\frac{1}{n+1}, \forall \gamma \notin \Gamma_{1}$. For every $0 \leq \xi<\eta$, write

$$
\Delta_{\xi}^{1}=\left(\eta^{n-1} \xi, \eta^{n-1}(\xi+1)\right]
$$

Let $Y_{1}$ be the subspace of $\mathbb{R}^{\eta^{n}}$ given by

$$
Y_{1}=\bigcap_{0 \leq \xi<\eta}\left\{y \in \mathbb{R}^{\eta^{n}}: y \text { is constant in } \Delta_{\xi}^{1}\right\} .
$$

Evidently $Y_{1}$ is isomorphic to $\mathbb{R}^{\eta}$.

We now claim that there exist $x_{1} \in X_{n}$ and $y_{1} \in Y_{1}$ such that $x_{1}=T^{-1}\left(y_{1}\right)$, $\left\|x_{1}\right\| \leq\left\|y_{1}\right\|=1$ and $\left\|x_{1}(\gamma)\right\|<\frac{1}{n+1}$ for every $\gamma \in \Gamma_{1}$.

In order to show this, we consider the operator $P_{\Gamma_{1}} T^{-1}: Y_{1} \rightarrow \sum_{\gamma \in \Gamma_{1}} X_{\gamma}$, where $P_{\Gamma_{1}}: \sum_{\gamma \in \Gamma} X_{\gamma} \rightarrow \sum_{\gamma \in \Gamma_{1}} X_{\gamma}$ is defined by $P_{\Gamma_{1}}\left(\left(x_{\gamma}\right)_{\gamma \in \Gamma}\right)=\left(x_{\gamma}\right)_{\gamma \in \Gamma_{1}}$.

Since $\mathbb{R}^{\eta} \hookrightarrow \sum_{\gamma \in \Gamma_{1}} X_{\gamma}$, it follows that $P_{\Gamma_{1}} T^{-1}$ is not an isomorphism of $Y_{1}$ into $\sum_{\gamma \in \Gamma_{1}} X_{\gamma}$. So there exists $y_{1} \in Y_{1}$ with $\left\|y_{1}\right\|=1$ such that $\left\|P_{\Gamma_{1}} T^{-1}\left(y_{1}\right)\right\|<\frac{1}{n+1}$. Put $x_{1}=T^{-1}\left(y_{1}\right)$. Hence

$$
\sup \left\{\left\|x_{1}(\gamma)\right\|: \gamma \in \Gamma_{1}\right\}=\left\|P_{\Gamma_{1}}\left(x_{1}\right)\right\|=\left\|P_{\Gamma_{1}} T^{-1}\left(y_{1}\right)\right\|<\frac{1}{n+1} .
$$

Finally, in view of $(2)$ we see that $\left\|x_{1}\right\| \leq\left\|T\left(x_{1}\right)\right\|=\left\|T T^{-1}\left(y_{1}\right)\right\|=\left\|y_{1}\right\|=1$, which establishes our claim.

Let $\xi_{1}$ be an ordinal such that $\left|y_{1}(t)\right| \geq 1 / 2$ for every $t \in \Delta_{\xi_{1}}^{1}$.

For the second step, let $\Gamma_{2}$ be a finite set containing $\Gamma_{1}$ such that $\left\|x_{1}(\gamma)\right\|<$ $\frac{1}{n+1}, \forall \gamma \notin \Gamma_{2}$. For every $0 \leq \xi<\eta$, write

$$
\Delta_{\xi}^{2}=\left(\eta^{n-1} \xi_{1}+\eta^{n-2} \xi, \eta^{n-1} \xi_{1}+\eta^{n-2}(\xi+1)\right] .
$$

Let $Y_{2}$ be the subspace of $\mathbb{R}^{\eta^{n}}$ given by

$$
Y_{2}=\bigcap_{0 \leq \xi<\eta}\left\{y \in \mathbb{R}^{\eta^{n}}: y \text { is constant in } \Delta_{\xi}^{2} \text { and } y(t)=0, \forall t \notin \Delta_{\xi_{1}}^{1}\right\} .
$$

It is simple to check that $Y_{2}$ is isomorphic to $\mathbb{R}^{\eta}$.

Arguing similarly as above, there exist $x_{2} \in X_{n}$ and $y_{2} \in Y_{2}$ such that $x_{2}=$ $T^{-1}\left(y_{2}\right),\left\|x_{2}\right\| \leq\left\|y_{2}\right\|=1$ and $\left\|x_{2}(\gamma)\right\|<\frac{1}{n+1}$ for every $\gamma \in \Gamma_{2}$.

Let $\xi_{2}$ be an ordinal such that $\left|y_{2}(t)\right| \geq \frac{1}{2}$ for every $t \in \Delta_{\xi_{2}}^{1}$.

Repeating this procedure $n$ times we will find

- $\Gamma_{1} \subset \Gamma_{2} \subset \ldots \subset \Gamma_{n} \subset \Gamma$,

- $a_{1}, a_{2}, \ldots, a_{n} \in \mathbb{R}$ with $\left|a_{i}\right| \geq 1 / 2$, for every $1 \leq i \leq n$,

- $x_{0}, x_{1}, \ldots, x_{n} \in X_{n}$, with $\left\|x_{i}\right\| \leq 1$, for every $1 \leq i \leq n$,

- $y_{0}=T\left(x_{0}\right), y_{1}=T\left(x_{1}\right), \ldots, y_{n}=T\left(x_{n}\right)$,

- $\Delta_{0}=\left[1, \eta^{n}\right] \supset \Delta_{1}=\Delta_{\xi_{1}}^{1} \supset \Delta_{2}=\Delta_{\xi_{2}}^{2} \supset \ldots \supset \Delta_{n}$, 
satisfying

$$
\begin{gathered}
\left\|x_{i}\right\| \leq 1 \text { and }\left\|x_{i}(\gamma)\right\|<\frac{1}{n+1} \text { whenever } \gamma \in \Gamma_{i} \text { or } \gamma \notin \Gamma_{i+1} \text { and } 1 \leq i \leq n, \\
y_{i}(t)=a_{i} \text { for every } t \in \Delta_{i} \text { and } 1 \leq i \leq n .
\end{gathered}
$$

Let $c_{i}$ be the sign of $a_{i}$ for every $1 \leq i \leq n$ and $z=\sum_{i=0}^{n} c_{i} x_{i}$.

Since $\Delta_{n}=\bigcap_{i=0}^{n} \Delta_{i}$, there exists $t_{0}$ belonging to all $\Delta_{i}, 0 \leq i \leq n$. Therefore by $(6)$,

$$
\|T(z)\|=\left\|\sum_{i=0}^{n} c_{i} y_{i}\right\| \geq\left|\sum_{i=0}^{n} c_{i} y_{i}\left(t_{0}\right)\right|=\sum_{i=0}^{n}\left|a_{i}\right| \geq \frac{n+1}{2} .
$$

On the other hand, put $A_{1}=\Gamma_{1}$ and $A_{i+1}=\Gamma_{i+1} \backslash \Gamma_{i}$ for $1 \leq i \leq n-1$. By (5) and by the fact that $\Gamma$ is the disjoint union of the sets $A_{i}, 1 \leq i \leq n$ and $\Gamma \backslash \Gamma_{n}$, we have

$$
\left\|x_{i}(t)\right\|<\frac{1}{n+1}
$$

for every $t \in \Gamma$ and for all indices $1 \leq i \leq n$ except at most one. Thus by the fact that $\left\|x_{i}\right\| \leq 1$ for every $1 \leq i \leq n$, we conclude that

$$
\|z\| \leq 1+n \frac{1}{n+1}<2 .
$$

Hence $\|T\| \geq\left\|T\left(\frac{z}{\|z\|}\right)\right\| \geq \frac{n+1}{4}$, which is absurd by (4) and the choice of $n$.

Proposition 2.13. Let $X$ be a Banach space having $M P$ and $\alpha$ an infinite initial ordinal. Suppose that $\mathbb{R}^{\alpha} \hookrightarrow c_{0}(\Gamma, X)^{\eta}$ for some set $\Gamma$ and $1 \leq \eta<\alpha$. Then $c_{0} \hookrightarrow X$.

Proof. If $\eta<\omega$, then the result follows immediately from [10, Lemma 2.2]. Next suppose that $\eta \geq \omega$. Since $\eta^{\omega^{2}}<\alpha$, by our hypothesis we see that

$$
\mathbb{R}^{\eta^{\omega^{2}}} \hookrightarrow c_{0}(\Gamma, X)^{\eta} \sim c_{0}(\Gamma, X) \hat{\hat{\otimes}} \mathbb{R}^{\eta} \sim c_{0}(\Gamma) \hat{\hat{\otimes}} X \hat{\hat{\otimes}} \mathbb{R}^{\eta} \sim c_{0}(\Gamma) \hat{\hat{\otimes}} X^{\eta} \sim c_{0}\left(\Gamma, X^{\eta}\right) .
$$

Then, according to Lemma 2.12 , there exists $1 \leq n<\omega$ such that

$$
\mathbb{R}^{\eta^{\omega}} \hookrightarrow X^{\eta} \oplus X^{\eta} \oplus \ldots \oplus X^{\eta} \sim(X \oplus X \oplus \ldots \oplus X)^{\eta} \sim\left(X^{n}\right)^{\eta} .
$$

Thus by Lemma 2.11, $c_{0} \hookrightarrow X^{n}$ and [20, Theorem 1] implies that $c_{0} \hookrightarrow X$.

\section{Proof of Theorem 1.1}

Before presenting the proof of Theorem 1.1 we still need to extend Lemma 2.9 and Proposition 2.10 for an arbitrary set $\Gamma$. The preceding proposition will play an important role in the proofs of these results.

Lemma 3.1. Let $X$ be a Banach space having $M P, \alpha$ a nondenumerable regular ordinal and $\Gamma$ a set. If $\mathbb{R}^{\alpha^{2}} \hookrightarrow c_{0}(\Gamma, X)^{\eta}$ for some $\eta<\alpha^{2}$, then $c_{0} \hookrightarrow X$.

Proof. As in Lemma 2.9, we distinguish two cases:

Case 1. $\eta<\alpha$. In this case, we have that $\mathbb{R}^{\alpha} \hookrightarrow c_{0}(\Gamma, X)^{\eta}$. Hence Proposition 2.13 implies that $c_{0} \hookrightarrow X$.

Case 2. $\alpha \leq \eta<\alpha^{2}$. Write $\eta=\alpha \xi+\theta$ for some ordinals $\xi<\alpha$ and $\theta<\alpha$. Notice that $\mathbb{R}^{\eta} \sim \mathbb{R}^{\alpha \xi}$ [16, Theorem 2]. Then,

$$
\mathbb{R}^{\alpha^{2}} \hookrightarrow c_{0}(\Gamma, X)^{\eta} \sim c_{0}(\Gamma, X) \hat{\hat{\otimes}} \mathbb{R}^{\eta} \sim c_{0}(\Gamma, X) \hat{\hat{\otimes}} \mathbb{R}^{\alpha \xi} \sim c_{0}(\Gamma, X)^{\alpha \xi} .
$$


Let $I$ and $J$ be two sets with $|I|=\bar{\alpha}$ and $|J|=\bar{\xi}$. Since $c_{0}(\Gamma, X)$ has MP [15, Corollary 5.3.4], we deduce by Lemma 2.4 and Proposition 2.8 that

$$
c_{0}(I) \sim \frac{\left[\mathbb{R}^{\alpha^{2}}\right]_{\alpha}}{c\left(\mathbb{R}^{\alpha^{2}}\right)} \hookrightarrow \frac{\left[c_{0}(\Gamma, X)^{\alpha \xi}\right]_{\alpha}}{c\left(c_{0}(\Gamma, X)^{\alpha \xi}\right)} \sim c_{0}(J, X)
$$

Then according to [10, Lemma 2.4] we conclude that $c_{0} \hookrightarrow X$.

Proposition 3.2. Let $\alpha$ be an initial ordinal and $\xi \leq \eta$ ordinals with $\bar{\xi}=\bar{\eta}=\bar{\alpha}$. Put $\alpha_{0}=\omega^{\omega}$ if $\alpha=\omega, \alpha_{0}=\alpha$ if $\alpha$ is a singular ordinal and $\alpha_{0}=\alpha^{2}$ if $\alpha$ is a nondenumerable regular ordinal. Suppose that $X$ is a Banach space having MP and containing no copy of $c_{0}$ and $\Gamma$ is a set. If $\mathbb{R}^{\eta} \hookrightarrow c_{0}(\Gamma, X)^{\xi}$ and $\alpha_{0} \leq \xi$, then $\mathbb{R}^{\eta} \hookrightarrow \mathbb{R}^{\xi}$

Proof. We introduce two sets of ordinals:

$I_{1}=\left\{\theta: \bar{\theta}=\bar{\alpha}, \alpha_{0} \leq \theta, \mathbb{R}^{\theta} \nrightarrow \mathbb{R}^{\gamma}, \forall \gamma<\theta\right\}$,

$I_{2}=\left\{\theta: \bar{\theta}=\bar{\alpha}, \alpha_{0} \leq \theta, \mathbb{R}^{\theta} \triangleleft c_{0}(\Gamma, X)^{\gamma}, \forall \gamma<\theta\right\}$.

According to [10, Lemma 2.2], Proposition 2.13 and Lemma 3.1, $\alpha_{0} \in I_{2}$. It is enough to proceed as in the proof of [10, Lemma 2.7] to complete the proof of this proposition.

Proof of Theorem 1.1. (1) Assume that $c_{0}(\Gamma, X)^{\xi} \sim c_{0}(\Gamma, X)^{\eta}$ and $\bar{\xi}<\bar{\eta}$. Let $\alpha$ be the initial ordinal of cardinality $\bar{\eta}$. Then $\mathbb{R}^{\alpha} \hookrightarrow c_{0}(\Gamma, X)^{\eta} \sim c_{0}(\Gamma, X)^{\xi}$ and by Proposition 2.13, $c_{0} \hookrightarrow X$, which is absurd.

For the sufficiency of the statements (2) and (3), see Remark 1.2. Next we will prove the necessity of these statements.

(2) Suppose thus that $c_{0}(\Gamma, X)^{\eta} \sim c_{0}(\Gamma, X)^{\xi}$. We distinguish two cases:

Case 1. $\alpha=\omega$. First assume that $\xi \geq \omega^{\omega}$. Then by hypothesis, $\mathbb{R}^{\eta} \hookrightarrow c_{0}(\Gamma, X)^{\eta} \sim$ $c_{0}(\Gamma, X)^{\xi}$. Thus Proposition 3.2 implies that $\mathbb{R}^{\eta} \hookrightarrow \mathbb{R}^{\xi}$ and by [16. Theorem 1], we have that $\eta<\xi^{\omega}$.

Now suppose that $\xi<\omega^{\omega}$. By [16, Theorem 1] and [1, Lemma 1.2] we conclude that $\mathbb{R}^{\xi} \sim \mathbb{R}^{\omega} \sim c_{0}$. Let $\mathbb{N}$ be the set of natural numbers. Then

$$
c_{0}(\Gamma \times \mathbb{N}, X) \sim c_{0}(\Gamma) \hat{\hat{\otimes}} X \hat{\hat{\otimes}} c_{0} \sim c_{0}(\Gamma) \hat{\hat{\otimes}} X \hat{\hat{\otimes}} \mathbb{R}^{\xi} \sim c_{0}(\Gamma, X)^{\xi} .
$$

Therefore, if $\eta>\xi^{\omega} \geq \omega^{\omega}$, we would have $\mathbb{R}^{\omega^{\omega}} \hookrightarrow \mathbb{R}^{\eta} \hookrightarrow c_{0}(\Gamma \times \mathbb{N}, X)$. Thus [10, Lemma 2.2] would imply that $c_{0} \hookrightarrow X$, which is absurd.

Case 2. $\alpha>\omega$. If $\eta>\xi^{\omega}$, then $\mathbb{R}^{\eta} \hookrightarrow c_{0}(\Gamma, X)^{\eta} \sim c_{0}(\Gamma, X)^{\xi}$. According to Proposition $3.2, \mathbb{R}^{\eta} \hookrightarrow \mathbb{R}^{\xi}$, which is absurd by [16, Theorem 1 ].

(3) Let $I$ and $J$ be two sets with $|I|=\bar{\xi}^{\prime}$ and $|J|=\bar{\eta}^{\prime}$. Since $c_{0}(\Gamma, X)$ has MP [15. Corollary 5.3.4], by Remark 2.3 and Proposition 2.8,

$$
c_{0}\left(I, c_{0}(\Gamma, X)\right) \sim \frac{\left[c_{0}(\Gamma, X)^{\alpha \xi^{\prime}}\right]_{\alpha}}{c\left(c_{0}(\Gamma, X)^{\alpha \xi^{\prime}}\right)} \sim \frac{\left[c_{0}(\Gamma, X)^{\alpha \eta^{\prime}}\right]_{\alpha}}{c\left(c_{0}(\Gamma, X)^{\alpha \eta^{\prime}}\right)} \sim c_{0}\left(J, c_{0}(\Gamma, X)\right) .
$$

Hence if $|\Gamma| \bar{\xi}^{\prime}$ and $|\Gamma| \bar{\eta}^{\prime}$ are finite, then $X^{|\Gamma| \bar{\xi}^{\prime}} \sim X^{|\Gamma|} \bar{\eta}^{\prime}$. On the other hand, if $|\Gamma| \bar{\xi}^{\prime}$ or $|\Gamma| \bar{\eta}^{\prime}$ is infinite, then applying [10, Lemma 2.4] we see that $|\Gamma| \bar{\xi}^{\prime}=|\Gamma| \bar{\eta}^{\prime}$.

(4) Suppose that $c_{0}(\Gamma, X)^{\xi} \sim c_{0}(\Gamma, X)^{\eta}$ with $\alpha \leq \xi<\alpha^{2} \leq \eta$. Then $\mathbb{R}^{\alpha^{2}} \hookrightarrow$ $\mathbb{R}^{\eta} \hookrightarrow c_{0}(\Gamma, X)^{\xi}$. Hence by Lemma 3.1, $c_{0} \hookrightarrow X$, and the proof is complete. 


\section{REFERENCES}

1. C. Bessaga, A. Pełczyński, Spaces of continuous functions. IV, Studia Math. XIX (1960), 53-62. MR0113132 (22:3971)

2. L. Burlando, On subspaces of direct sums of infinite sequences of Banach spaces, Atti Accad. Ligure Sci. Lett. 46 (1989), 96-105 (1990). MR1098789 (92b:46019)

3. A. Defant, K. Floret, Tensor norms and operator ideals, Math. Studies, 176, North-Holland, Amsterdam (1993). MR.1209438 (94e:46130)

4. A. Defant, J. A. López-Molina, M. J. Rivera, On Pitt's theorem for operators between scalar and vector-valued quasi-Banach sequence spaces, Monatsh. Math. 130 (2000), 7-18. MR 1762060 (2001e:46009)

5. J. Diestel, J.J. Uhl, Jr., Vector measures, Mathematical Surveys, 15, Amer. Math. Soc., Providence, RI (1977). MR0453964 (56:12216)

6. G. A. Edgar, Measurability in a Banach space. II, Indiana Univ. Math. J. 28 (1977), 559-579. MR:542944 (81d:28016)

7. E. M. Galego, How to generate new Banach spaces non-isomorphic to their Cartesian squares, Bull. Polish Acad. Sci. Math. 47 (1999), 1, 21-25. MR1685684 (2001b:46015)

8. E. M. Galego, Banach spaces of continuous vector-valued functions of ordinals, Proc. Edinb. Math. Soc. (2) 44 (2001), 1, 49-62. MR1879208 (2002k:46064)

9. E. M. Galego, On subspaces and quotients of Banach spaces $C(K, X)$, Monatsh. Math. 136 (2002), 2, 87-97. MR.1914222 (2003g:46038)

10. E. M. Galego, On isomorphic classifications of compact operators, Proc. Amer. Math. Soc. 137 (2009), 3335-3342.

11. R. J. Gardner, W. F. Pfeffer, Borel measures, Handbook of set-theoretic topology, NorthHolland, Amsterdam (1984), 961-1043. MR776641 (86c:28031)

12. S. P. Gul'ko, A. V. Os'kin, Isomorphic classification of spaces of continuous functions on totally ordered bicompacta, Functional Anal. Appl. 9 (1975), 1, 56-57. MR0377489 (51:13661)

13. W. B. Johnson, J. Lindenstrauss, Basic concepts in the geometry of Banach spaces, Handbook of the geometry of Banach spaces, North-Holland, Amsterdam (2001), 1-84. MR 1863689 (2003f:46013)

14. A. Kanamori, M. Magidor, The evolution of large cardinal axioms in set theory, Higher set theory (Proc. Conf., Math. Forschungsinst., Oberwolfach, 1977), Lecture Notes in Math., 669, Springer, Berlin (1978), 99-275. MR520190 (80b:03083)

15. T. Kappeler, Banach spaces with the condition of Mazur, Math. Z. 191 (1986), 623-631. MR $832820(87 \mathrm{~h}: 46040)$

16. S. V. Kislyakov, Classification of spaces of continuous functions of ordinals, Siberian Math. J. 16 (1975), 2, 226-231.

17. M. A. Labbé, Isomorphisms of continuous function spaces, Studia Math. LII (1974/75), 221231. MR0390737 (52:11560)

18. D. Leung, On Banach spaces with Mazur's property, Glasgow Math. J. 33 (1991), 51-54. MR.1089953 (92b:46021)

19. J. Lindenstrauss, L. Tzafriri, Classical Banach spaces. I. Sequence spaces, Springer-Verlag, Berlin-New York (1977). MR0500056 (58:17766)

20. C. Samuel, Sur la reproductibilité des espaces $l_{p}$, Math. Scand. 45 (1979), 103-117. MR567436 (81e:46062)

21. C. Samuel, Sur les sous-espaces de $l_{p} \hat{\otimes} l_{q}$, Math. Scand. 47 (1980), 247-250. MR612698 (82i:46029)

22. C. Samuel, On spaces of operators on $C(Q)$ spaces ( $Q$ countable metric space), Proc. Amer. Math. Soc. 137 (2009), 3, 965-970. MR2457436

23. Z. Semadeni, Banach spaces non-isomorphic to their Cartesian squares. II, Bull. Acad. Polon. Sci. Sér. Sci. Math. Astr. Phys. 8 (1960), 81-84. MR0115074 (22:5877)

24. A. Wilansky, Mazur spaces, Internat. J. Math. Math. Sci. 4 (1981), 39-53. MR606656 (82f:46001)

25. X. P. Xue, Y. J. Li, Q. Y. Bu, Some properties of the injective tensor product of Banach spaces, Acta Math. Sinica (English series) 23 (2007), 9, 1697-1706. MR2326510

Department of Mathematics, University of São Paulo, São Paulo, Brazil 05508-090 E-mail address: eloi@ime.usp.br 